\title{
Sonographic Detection of Monoamniotic Twins
}

\author{
Caroline E. Blane, MD, Michael A. DiPietro, MD, Mary Z. Johnson, MD, \\ Susan J. White, MD, Grace I. Louwsma, RN, and Joan E. Hamman, RN
}

\begin{abstract}
The true incidence of monoamniotic twinning has been almost impossible to determine accurately because it requires closer inspection of the membranes and placenta at delivery than is usually performed. Sonography of 3440 patients presenting for genetic amniocentesis identified 39 twin gestations, one of which was monoamniotic $(0.026 \%)$. Prospectively with ultrasound, four of the twin gestations had been thought to be monoamniotic. A more recent case of suspected monoamniotic twinning revealed two sacs when newer computer-based real-time equipment with variable focusing capability and improved spatial resolution was used. Sonographic diagnosis of monoamniotic twinning must be accurate since it identifies patients at higher risk for cord accidents. These patients need obstetrical care appropriate for a pregnancy at high risk. In addition, failure to identify a second sac that could harbor a chromosomally abnormal fetus has both medical and legal implications. State-of-the-art ultrasound equipment and attention to detail is required. Indexing Words: Monoamniotic twins - Ultrasound
\end{abstract}

The incidence of monoamniotic twinning has been estimated to be between 1 in 6000 and 1 in 60,000 pregnancies. ${ }^{1}$ However, the true incidence is almost impossible to determine accurately because it requires closer inspection of the membranes and placenta than is usually performed at the time of delivery of twins. ${ }^{1}$

Ultrasound is used to diagnose multiple gestations in early pregnancy. It has been reported that the number of fetuses can be easily identified as well as the number of amniotic sacs. ${ }^{2,3}$ Sonographic evaluation in some obstetrical cases may be incomplete and information regarding the number of amniotic sacs not included. However, when the gravid uterus is examined sonographically prior to a genetic amniocentesis, identification of the number of sacs and the exact location of the membranes is of extreme importance. Most genetic amniocenteses are now carried out to identify chromosomal abnormalities when maternal age is greater than 35 years. In the case of twins,

From the Departments of Radiology (CEB, SJW, MAD) and Obstetrics and Gynecology (MZJ, GIL, JEH), The University of Michigan Medical Center, Ann Arbor, Michigan. For reprints contact Caroline Blane, MD, Department of Radiology, Section of Pediatric Radiology, University of Michigan Hospitals, C.S. Mott Children's Hospital, Room C3123/0252, Ann Arbor, Michigan 48109-0252. information is sought on each twin, requiring amniocentesis of each sac.

\section{MATERIALS AND METHODS}

Between 1975 and 1983 there have been 3440 patients seen for amniocentesis with an ultrasound examination immediately before the procedure. Sonography of the first 1019 patients was performed with a static B-mode scanner.'Since 1979 real-time scanning with $3.5-$ or $5-\mathrm{MHz}$ mechanical sector scanner transducer has been included in each study. The records were carefully reviewed for the occurrence of twin gestation and of suspected monoamniotic twinning. Detailed information on the placenta and amniotic and chorionic membranes was requested from the delivering obstetrician in each case of suspected monoamniotic twinning.

\section{RESULTS}

The vast majority ( $>80 \%$ ) of amniocenteses were done for maternal age over 35 years. In the population of 3440 there were 39 patients with twin gestations ( 1 in 88 pregnancies). Of these, sonography had identified four patients in whom no separating membrane could be found and that were thought to be monoamniotic twins. All four were 
seen since 1979 and had careful, detailed realtime sonography with equipment that was then considered state-of-the-art. The four women with suspected monoamniotic twins were aged 36-39 years. Three patients were primigravidas and one woman had one previous child. A single amniotic fluid sample was obtained in all cases. All four amniotic samples had normal karyotypes. Examination of the placenta at delivery revealed that three of the patients had diamniotic, monochorionic twin gestations. The sole monoamniotic, monochorionic gestation was in a 39-year-old primigravida who delivered two healthy boys at term.

In one subsequent case a monoamniotic twin gestation was suspected sonographically with the real-time scanner used in this study (Fig. 1). However, a repeat sonogram obtained with a new stateof-the-art, computer-based, real-time, variably electronically focused scanner with a 5-MH2 transducer clearly revealed the amniotic membrane separating the twins (Fig. 2). The patient then had an amniocentesis on each amniotic sac.

\section{DISCUSSION}

In the general population the incidence of twinning is 1 in 80 pregnancies. ${ }^{1}$ Several mechanisms are involved in twin gestation. The most common is dizygotic twinning in which two ova are fertilized in a single ovulatory cycle and two separate gestational sacs develop. ${ }^{1,2}$ Monozygotic twinning can occur in two ways. The early blastomeres can separate into two separate gestational sacs or the inner cell mass can later duplicate, resulting in twin embryos in a single chorion (monochorionic) and a single gestational monoamniotic sac. ${ }^{1,2}$ This latter mechanism is thought to be rare, accounting for only 1 in 6000 to 1 in 60,000 pregnancies. ${ }^{1}$ Of twin pregnancies this would account for only 1 in 70 to 1 in $700 .{ }^{1}$ However, many monoamniotic twins may be undetected and unreported because close inspection of the placenta and membranes is not always carried out at the time of delivery. ${ }^{1}$

Our carefully screened population revealed an occurrence of monoamniotic twins of 1 in 39 twin gestations or 1 in 3440 pregnancies $(0.029 \%)$. Our population was definitely older than the general population of pregnant mothers, the majority being greater than 35 years of age. However, our population is typical of that undergoing amniocentesis and should approximate the frequency of twins in that population. All multiple gestations are at higher risk for prenatal mortality mainly due to the higher risk of premature delivery. ${ }^{3}$ However,

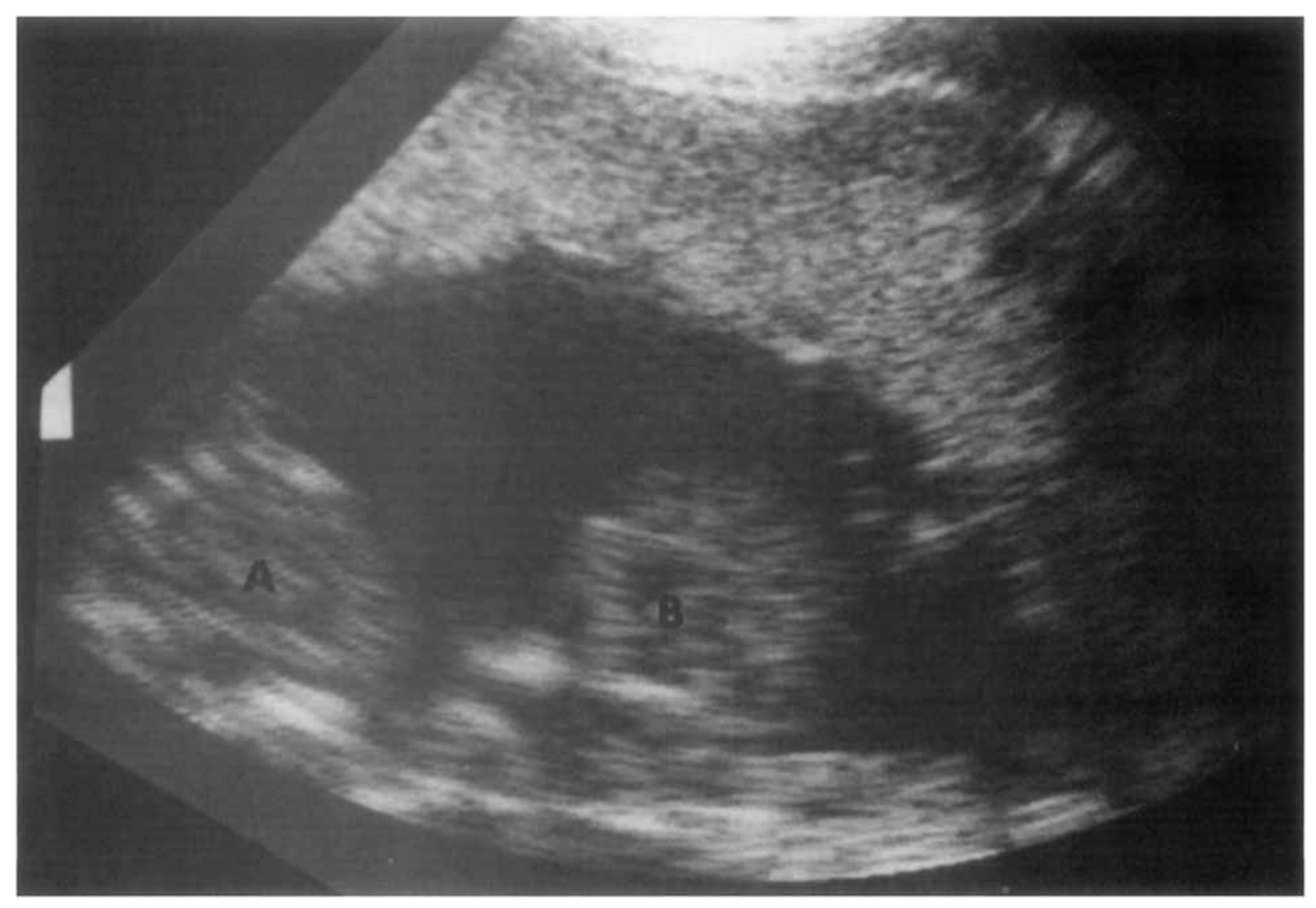

FIGURE 1. Real-time sonography fails to reveal a membrane separating twin A (body of A) from twin B (body of B). 


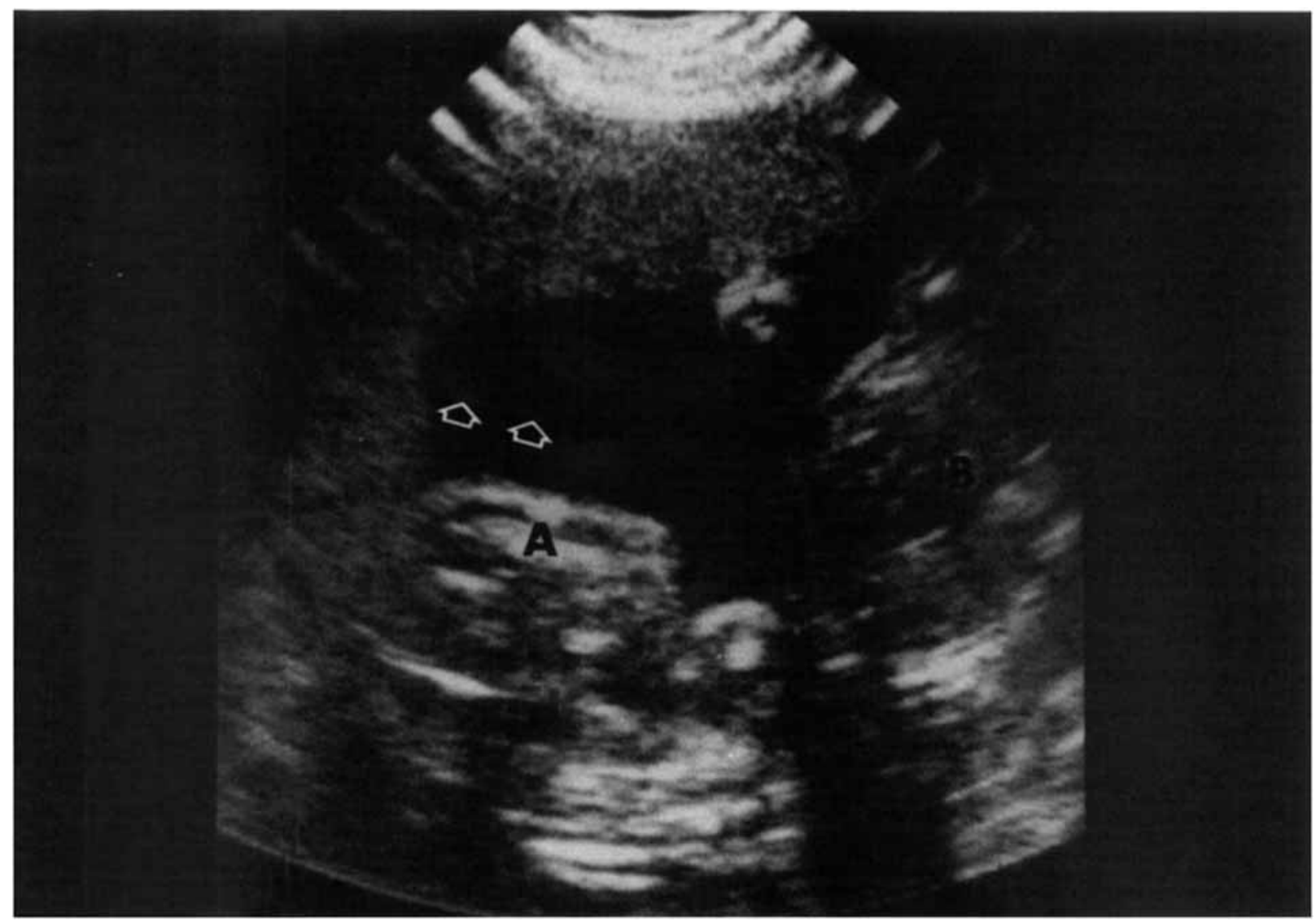

FIGURE 2. Computer-based real-time equipment with variable focusing capability demonstrates the membrane (arrows) above twin A.

in monoamniotic twinning an additional complication exists: knotting of the umbilical cords. Case reports of unusual cord complications can be found in the literature, ${ }^{4,5}$ including a recent sonographic diagnosis of this complication. ${ }^{6}$ Thus, identifying monoamniotic twins is important beyond the need for complete karyotype determination at amniocentesis.

Prospectively four cases of monoamniotic twinning were suspected. Examination of the placenta at delivery showed that only one was truly monoamniotic. The new computer-based, real-time, mechanical sector scanners with variable electronic focusing capability have improved spatial resolution. The amniotic membrane is thinner than the spatial resolution available with older ultrasonic equipment. Since failure to identify a second sac that could harbor a chromosomally abnormal fetus would suggest the false diagnosis of monoamniotic twins, the new, higher-resolution equipment should be used. Real-time sonography facilitates identification of the number of gestational sacs, but attention to detail and state-of-the-art equipment is needed.

\section{ACKNOWLEDGMENTS}

The authors thank Drs. John O'Sullivan, Robert Saig, D. C. Gustafson, and K. Cowles for their help in documenting the pathology.

\section{REFERENCES}

1. Salerno LJ: Monoamniotic twinning: A survey of the American literature since 1935 with a report of four new cases. Obstet Gynecol 14:205, 1959.

2. Mantoni M, Pedersen JF: Case report-monoamniotic twins diagnosed by ultrasound in the first trimester. Acta Obstet Gynecol Scand 59:551, 1980.

3. Milne B: Problems in ultrasonic monitoring of multiple pregnancies. $\mathrm{Br} J$ Radiol 53:85, 1980.

4. Golan A, Amit A, Baram A, et al: Unusual cord intertwining in monoamniotic twins. Aust $\mathrm{NZ} \mathrm{JOb-}$ stet Gynaecol 22:165, 1982.

5. McLeod FN, McCoy DR: Monoamniotic twins with an unusual cord complication-case report. $\mathrm{BrJOb}$ stet Gynaecol 88:774, 1981.

6. Nyberg DA, Filly RA, Golbus MS, et al: Case reports-entangled umbilical cords: A sign of monoamniotic twins. J Ultrasound Med 3:29, 1984. 